\title{
On the growth estimates of entire functions of double complex variables
}

\author{
Sanjib Kumar Datta ${ }^{1}$, Tanmay Biswas ${ }^{2}$ \\ ${ }^{1}$ Department of Mathematics, University of Kalyani, P.O.-Kalyani, Dist-Nadia, PIN- 741235, West Bengal, India \\ ${ }^{2}$ Rajbari, Rabindrapalli, R. N. Tagore Road, P.O.-Krishnagar, Dist-Nadia, PIN-741101, West Bengal, India
}

Received: 25 June 2016, Accepted: 7 December 2016

Published online: 16 August 2017.

\begin{abstract}
Recently Datta et al. [6] introduced the idea of relative type and relative weak type of entire functions of two complex variables with respect to another entire function of two complex variables and prove some related growth properties of it. In this paper, further we study some growth properties of entire functions of two complex variables on the basis of their relative types and relative weak types as introduced by Datta et al [6].
\end{abstract}

Keywords: Entire function of two complex variables, order (lower order) of entire function of two complex variables, relative order (relative lower order) of entire functions of two complex variable, relative type (relative weak type) of two complex variables.

\section{Introduction, definitions and notations}

Suppose $f$ be an entire function of two complex variables holomorphic in the closed polydisc

$$
U=\left\{\left(z_{1}, z_{2}\right):\left|z_{i}\right| \leq r_{i}, i=1,2 \text { for all } r_{1} \geq 0, r_{2} \geq 0\right\}
$$

and $M_{f}\left(r_{1}, r_{2}\right)=\max \left\{\left|f\left(z_{1}, z_{2}\right)\right|:\left|z_{i}\right| \leq r_{i}, i=1,2\right\}$. Then in the light of maximum principal and Hartogs's theorem \{[7], p.2, p.51\}, $M_{f}\left(r_{1}, r_{2}\right)$ is an increasing function of $r_{1}, r_{2}$. For any two entire functions $f$ and $g$ of two complex variables, the ratio $\frac{M_{f}\left(r_{1}, r_{2}\right)}{M_{g}\left(r_{1}, r_{2}\right)}$ as $r_{1}, r_{2} \rightarrow \infty$ is called the growth of $f$ with respect to $g$. Taking this into account, the following definition is well known.

Definition 1. ([7], p.339, see also [1]) The order ${ }_{v_{2}} \rho_{f}$ and lower order ${ }_{v_{2}} \lambda_{f}$ of an entire function $f\left(z_{1}, z_{2}\right)$ are defined as

$$
{ }_{v_{2}} \rho_{f}=\limsup _{r_{1}, r_{2} \rightarrow \infty} \frac{\log \log M_{f}\left(r_{1}, r_{2}\right)}{\log M_{\exp \left(z_{1} z_{2}\right)}\left(r_{1}, r_{2}\right)}=\limsup _{r_{1}, r_{2} \rightarrow \infty} \frac{\log \log M_{f}\left(r_{1}, r_{2}\right)}{\log M_{\exp \left(z_{1} z_{2}\right)}\left(r_{1}, r_{2}\right)}
$$

and

$$
{ }_{v_{2}} \lambda_{f}=\liminf _{r_{1}, r_{2} \rightarrow \infty} \frac{\log \log M_{f}\left(r_{1}, r_{2}\right)}{\log M_{\exp \left(z_{1} z_{2}\right)}\left(r_{1}, r_{2}\right)}=\liminf _{r_{1}, r_{2} \rightarrow \infty} \frac{\log \log M_{f}\left(r_{1}, r_{2}\right)}{\log M_{\exp \left(z_{1} z_{2}\right)}\left(r_{1}, r_{2}\right)} .
$$

We see that the order ${ }_{v_{2}} \rho_{f}$ and lower order ${ }_{v_{2}} \lambda_{f}$ of an entire function $f\left(z_{1}, z_{2}\right)$ is defined in terms of the growth of $f\left(z_{1}, z_{2}\right)$ with respect to the exponential function $\exp \left(z_{1} z_{2}\right)$.

The rate of growth of an entire function generally depends upon the order (lower order) of it. The entire function with higher order is of faster growth than that of lesser order. But if orders of two entire functions are the same, then it is impossible to detect the function with faster growth. In that case, it is necessary to compute another class of growth indicators of entire functions called their types and thus one can define type of an entire function $f\left(z_{1}, z_{2}\right)$ denoted by $v_{2} \sigma_{f}$ in the following way. 
Definition 2. [6] The type ${ }_{v_{2}} \sigma_{f}$ of an entire function $f\left(z_{1}, z_{2}\right)$ is defined as

$$
{ }_{v_{2}} \sigma_{f}=\limsup _{r_{1}, r_{2} \rightarrow \infty} \frac{\log M_{f}\left(r_{1}, r_{2}\right)}{\left[r_{1} r_{2}\right]^{v_{2}} \rho_{f}}, 0<{ }_{v_{2}} \rho_{f}<\infty .
$$

Similarly, the lower type ${ }_{v_{2}} \bar{\sigma}_{f}$ of an entire function $f\left(z_{1}, z_{2}\right)$ may be defined as

$$
{ }_{v_{2}} \bar{\sigma}_{f}=\liminf _{r_{1}, r_{2} \rightarrow \infty} \frac{\log M_{f}\left(r_{1}, r_{2}\right)}{\left[r_{1} r_{2}\right]^{v_{2}} \rho_{f}}, 0<{ }_{v_{2}} \rho_{f}<\infty .
$$

Similarly in order to determine the relative growth of two entire functions of two complex variables having same non zero finite lower order one may introduce the concept of weak type $v_{2} \tau_{f}$ of $f\left(z_{1}, z_{2}\right)$ of finite positive lower order ${ }_{v_{2}} \lambda_{f}$ which is as follows.

Definition 3. [6] The weak type ${ }_{v_{2}} \tau_{f}$ of an entire function $f\left(z_{1}, z_{2}\right)$ of finite positive lower order ${ }_{v_{2}} \lambda_{f}$ is defined by

$$
{ }_{v_{2}} \tau_{f}=\liminf _{r_{1}, r_{2} \rightarrow \infty} \frac{\log M_{f}\left(r_{1}, r_{2}\right)}{\left[r_{1} r_{2}\right]^{v_{2}} \lambda_{f}}, 0<{ }_{v_{2}} \lambda_{f}<\infty .
$$

Likewise, one may define the growth indicator $v_{2} \bar{\tau}_{f}$ of an entire function $f\left(z_{1}, z_{2}\right)$ of finite positive lower order ${ }_{v_{2}} \lambda_{f}$ in the following way.

$$
v_{2} \bar{\tau}_{f}=\limsup _{r_{1}, r_{2} \rightarrow \infty} \frac{\log M_{f}\left(r_{1}, r_{2}\right)}{\left[r_{1} r_{2}\right]^{v_{2}} \lambda_{f}}, 0<{ }_{v_{2}} \lambda_{f}<\infty .
$$

Bernal (see [2], [3]) introduced the definition of relative order between two entire functions of single variable. During the past decades, several authors ( see [8],[9],[10],[11]) made closed investigations on the properties of relative order of entire functions of single variable. Using the idea of Bernal's relative order (see [2], [3]) of entire functions of single variable, Banerjee and Datta [4] introduced the definition of relative order of entire functions of two complex variables to avoid comparing growth just with $\exp \left(z_{1} z_{2}\right)$ which is as follows.

$$
{ }_{v_{2}} \rho_{g}(f)=\inf \left\{\mu>0: M_{f}\left(r_{1}, r_{2}\right)<M_{g}\left(r_{1}^{\mu}, r_{2}^{\mu}\right) ; r_{1} \geq R(\mu), r_{2} \geq R(\mu)\right\}=\limsup _{r_{1}, r_{2} \rightarrow \infty} \frac{\log M_{g}^{-1} M_{f}\left(r_{1}, r_{2}\right)}{\log \left(r_{1} r_{2}\right)}
$$

where $g$ is also an entire function holomorphic in the closed polydisc

$$
U=\left\{\left(z_{1}, z_{2}\right):\left|z_{i}\right| \leq r_{i}, i=1,2 \text { for all } r_{1} \geq 0, r_{2} \geq 0\right\}
$$

and the definition coincides with the classical one [4] if $g\left(z_{1}, z_{2}\right)=\exp \left(z_{1} z_{2}\right)$.

Like wise, one can define the relative lower order of $f$ with respect to $g$ denoted by ${ }_{v_{2}} \lambda_{g}(f)$ as follows :

$$
{ }_{v_{2}} \lambda_{g}(f)=\liminf _{r_{1}, r_{2} \rightarrow \infty} \frac{\log M_{g}^{-1} M_{f}\left(r_{1}, r_{2}\right)}{\log \left(r_{1} r_{2}\right)} .
$$

Now in the case of relative order of entire functions of two complex variables, it therefore seems reasonable to define suitably the relative type and relative weak type respectively in order to compare the relative growth of two entire functions of two complex variables having same non zero finite relative order or relative lower order with respect to another entire function of two complex variables. Recently Datta et al [6] introduced such definitions which are as follows.

Definition 4. [6] Let $f\left(z_{1}, z_{2}\right)$ and $g\left(z_{1}, z_{2}\right)$ be any two entire functions such that $0<{ }_{v_{2}} \rho_{g}(f)<\infty$. Then the relative type ${ }_{v_{2}} \sigma_{g}(f)$ of $f\left(z_{1}, z_{2}\right)$ with respect to $g\left(z_{1}, z_{2}\right)$ is defined as.

$$
{ }_{v_{2}} \sigma_{g}(f)=\inf \left\{k>0: M_{f}\left(r_{1}, r_{2}\right)<M_{g}\left(k r_{1}^{v_{2}} \rho_{g}(f), k r_{2}^{v_{2} \rho_{g}(f)}\right) \quad \text { for all sufficiently large values of } r_{1} \text { and } r_{2}\right\} \text {. }
$$


Equivalent formula for ${ }_{v_{2}} \sigma_{g}(f)$ is

$$
{ }_{v_{2}} \sigma_{g}(f)=\limsup _{r_{1}, r_{2} \rightarrow \infty} \frac{M_{g}^{-1} M_{f}\left(r_{1}, r_{2}\right)}{\left[r_{1} r_{2}\right]^{v_{2}} \rho_{g}(f)} .
$$

Likewise, one can define the relative lower type of an entire function $f\left(z_{1}, z_{2}\right)$ with respect to an entire function $g\left(z_{1}, z_{2}\right)$ denoted by ${ }_{v_{2}} \bar{\sigma}_{g}(f)$ as follows

$$
v_{2} \bar{\sigma}_{g}(f)=\liminf _{r_{1}, r_{2} \rightarrow \infty} \frac{M_{g}^{-1} M_{f}\left(r_{1}, r_{2}\right)}{\left[r_{1} r_{2}\right]^{v_{2}} \rho_{g}(f)}, 0<v_{2} \rho_{g}(f)<\infty .
$$

Definition 5. [6] The relative weak type $v_{2} \tau_{g}(f)$ of an entire function $f\left(z_{1}, z_{2}\right)$ with respect to another entire function $g\left(z_{1}, z_{2}\right)$ having finite positive relative lower order ${ }_{v_{2}} \lambda_{g}(f)$ is defined as

$$
{ }_{v_{2}} \tau_{g}(f)=\liminf _{r_{1}, r_{2} \rightarrow \infty} \frac{M_{g}^{-1} M_{f}\left(r_{1}, r_{2}\right)}{\left[r_{1} r_{2}\right]^{{ }^{v_{2}}} \lambda_{g}(f)} \text {. }
$$

Also one may define the growth indicator ${ }_{v_{2}} \bar{\tau}_{g}(f)$ of an entire function $f$ with respect to an entire function $g$ in the following way

$$
{ }_{v_{2}} \bar{\tau}_{g}(f)=\limsup _{r_{1}, r_{2} \rightarrow \infty} \frac{M_{g}^{-1} M_{f}\left(r_{1}, r_{2}\right)}{\left[r_{1} r_{2}\right]^{v_{2}} \lambda_{g}(f)}, 0<{ }_{v_{2}} \lambda_{g}(f)<\infty .
$$

Considering $g\left(z_{1}, z_{2}\right)=\exp \left(z_{1} z_{2}\right)$ one may easily verify that Definition 4 and Definition 5 coincide with Definition 2 and Definition 3 respectively.

In the paper we investigate some relative growth properties of entire functions of two complex variables with respect to another entire function of two complex variables on the basis of relative type and relative weak type of two complex variables. We do not explain the standard definitions and notations in the theory of entire functions as those are available in [7].

\section{Lemma}

In this section we present a lemma due to Datta et al. [5].

Lemma 1. [5] Let $f$ and $g$ be any two entire functions of two complex variables with

$$
0 \leq_{v_{2}} \lambda_{f} \leq_{v_{2}} \rho_{f}<\infty \text { and } 0 \leq_{v_{2}} \lambda_{g} \leq_{v_{2}} \rho_{g}<\infty
$$

Then

$$
\frac{v_{2} \lambda_{f}}{{ }_{v_{2}} \rho_{g}} \leq{ }_{v_{2}} \lambda_{g}(f) \leq \min \left\{\frac{v_{2} \lambda_{f}}{{ }_{v_{2}} \lambda_{g}}, \frac{v_{2} \rho_{f}}{{ }_{v_{2}} \rho_{g}}\right\} \leq \max \left\{\frac{{ }_{v_{2}} \lambda_{f}}{{ }_{v_{2}} \lambda_{g}}, \frac{v_{2} \rho_{f}}{{ }_{v_{2}} \rho_{g}}\right\} \leq{ }_{v_{2}} \rho_{g}(f) \leq \frac{v_{2} \rho_{f}}{v_{2} \lambda_{g}}
$$

\section{Theorems}

In this section we present the main results of the paper.

Theorem 1. Let $f\left(z_{1}, z_{2}\right)$ and $g\left(z_{1}, z_{2}\right)$ be any two entire functions with $0 \leq{ }_{v_{2}} \lambda_{f} \leq{ }_{v_{2}} \rho_{f}<\infty$ and $0 \leq{ }_{v_{2}} \lambda_{g} \leq{ }_{v_{2}} \rho_{g}<\infty$. Then

$$
\max \left\{\left[\frac{v_{2} \bar{\sigma}_{f}}{{ }_{v_{2}} \tau_{g}}\right]^{\frac{1}{v_{2} \lambda_{g}}},\left[\frac{v_{2} \sigma_{f}}{v_{2} \bar{\tau}_{g}}\right]^{\frac{1}{v_{2} \lambda_{g}}}\right\} \leq v_{2} \sigma_{g}(f) \leq \min \left\{\left[\frac{v_{2} \bar{\tau}_{f}}{v_{2} \tau_{g}}\right]^{\frac{1}{v_{2} \lambda_{g}}},\left[\frac{v_{2} \sigma_{f}}{v_{2} \bar{\sigma}_{g}}\right]^{\frac{1}{v_{2} \rho_{g}}},\left[\frac{v_{2} \bar{\tau}_{f}}{v_{2} \bar{\sigma}_{g}}\right]^{\frac{1}{v_{2} \rho_{g}}}\right\}
$$

and

$$
\left[\frac{v_{2} \bar{\sigma}_{f}}{v_{2} \bar{\tau}_{g}}\right]^{\frac{1}{v_{2} \lambda_{g}}} \leq v_{2} \bar{\sigma}_{g}(f) \leq \min \left\{\begin{array}{l}
{\left[\frac{v_{2} \bar{\sigma}_{f}}{v_{2} \bar{\sigma}_{g}}\right]^{\frac{1}{v_{2} \rho_{g}}},\left[\frac{v_{2} \sigma_{f}}{v_{2} \sigma_{g}}\right]^{\frac{1}{v_{2} \rho_{g}}},\left[\frac{v_{2} \tau_{f}}{v_{2} \tau_{g}}\right]^{\frac{1}{v_{2} \lambda_{g}}},} \\
{\left[\frac{v_{2}}{v_{f}} \bar{\tau}_{f}\right]^{\frac{1}{v_{2} \lambda_{g}}},\left[\frac{v_{2} \bar{\tau}_{f}}{v_{2} \sigma_{g}}\right]^{\frac{1}{v_{2} \rho_{g}}},\left[\frac{v_{2} \tau_{f}}{v_{2} \bar{\sigma}_{g}}\right]^{\frac{1}{v_{2} \rho_{g}}}}
\end{array}\right\}
$$


Proof. From the definitions of ${ }_{v_{2}} \sigma_{f}$ and ${ }_{v_{2}} \bar{\sigma}_{f}$, we have for all sufficiently large values of $r_{1}, r_{2}$ that

$$
\begin{aligned}
& M_{f}\left(r_{1}, r_{2}\right) \leq \exp \left\{\left(v_{2} \sigma_{f}+\varepsilon\right)\left[r_{1} r_{2}\right]^{v_{2}} \rho_{f}\right\} \\
& M_{f}\left(r_{1}, r_{2}\right) \geq \exp \left\{\left(v_{2} \bar{\sigma}_{f}-\varepsilon\right)\left[r_{1} r_{2}\right]^{v_{2}} \rho_{f}\right\}
\end{aligned}
$$

and also for a sequence of values of $r_{1}, r_{2}$ tending to infinity, we get that

$$
\begin{aligned}
& M_{f}\left(r_{1}, r_{2}\right) \geq \exp \left\{\left(v_{2} \sigma_{f}-\varepsilon\right)\left[r_{1} r_{2}\right]^{v_{2} \rho_{f}}\right\}, \\
& M_{f}\left(r_{1}, r_{2}\right) \leq \exp \left\{\left(v_{2} \bar{\sigma}_{f}+\varepsilon\right)\left[r_{1} r_{2}\right]^{v_{2}} \rho_{f}\right\} .
\end{aligned}
$$

Similarly from the definitions of ${ }_{v_{2}} \sigma_{g}$ and $v_{2} \bar{\sigma}_{f}$, it follows for all sufficiently large values of $r_{1}, r_{2}$ that

$$
M_{g}\left(r_{1}, r_{2}\right) \leq \exp \left\{\left({ }_{v_{2}} \sigma_{g}+\varepsilon\right)\left[r_{1} r_{2}\right]^{v_{2} \rho_{g}}\right\}
$$

Thus

$$
\left[r_{1} r_{2}\right] \leq M_{g}^{-1}\left[\exp \left\{\left(v_{2} \sigma_{g}+\varepsilon\right)\left[r_{1} r_{2}\right]^{v_{2} \rho_{g}}\right\}\right]
$$

and

$$
M_{g}^{-1}\left(r_{1}, r_{2}\right) \geq\left[\left(\frac{\log \left(r_{1} r_{2}\right)}{\left(v_{2} \sigma_{g}+\varepsilon\right)}\right)^{\frac{1}{v_{2} \rho_{g}}}\right]
$$

$$
M_{g}\left(r_{1}, r_{2}\right) \geq \exp \left\{\left(v_{2} \bar{\sigma}_{g}-\varepsilon\right)\left[r_{1} r_{2}\right]^{v_{2} \rho_{g}}\right\}
$$

Thus

$$
\left[r_{1} r_{2}\right] \geq M_{g}^{-1}\left[\exp \left\{\left(v_{2} \bar{\sigma}_{g}-\varepsilon\right)\left[r_{1} r_{2}\right]^{v_{2} \rho_{g}}\right\}\right]
$$

and

$$
M_{g}^{-1}\left(r_{1}, r_{2}\right) \leq\left[\left(\frac{\log \left(r_{1} r_{2}\right)}{\left(v_{2} \bar{\sigma}_{g}-\varepsilon\right)}\right)^{\frac{1}{v_{2} \rho_{g}}}\right]
$$

and for a sequence of values of $r_{1}, r_{2}$ tending to infinity, we obtain that

$$
M_{g}\left(r_{1}, r_{2}\right) \geq \exp \left\{\left({ }_{v_{2}} \sigma_{g}-\varepsilon\right)\left[r_{1} r_{2}\right]^{v_{2} \rho_{g}}\right\} .
$$

Thus

$$
\left[r_{1} r_{2}\right] \geq M_{g}^{-1}\left[\exp \left\{\left(v_{2} \sigma_{g}-\varepsilon\right)\left[r_{1} r_{2}\right]^{v_{2} \rho_{g}}\right\}\right]
$$

and

$$
\begin{gathered}
M_{g}^{-1}\left(r_{1}, r_{2}\right) \leq\left[\left(\frac{\log \left(r_{1} r_{2}\right)}{\left(v_{2} \sigma_{g}-\varepsilon\right)}\right)^{\frac{1}{v_{2} \rho_{g}}}\right] . \\
M_{g}\left(r_{1}, r_{2}\right) \leq \exp \left\{\left(v_{2} \bar{\sigma}_{g}+\varepsilon\right)\left[r_{1} r_{2}\right]^{{ }^{\nu_{2}} \rho_{g}}\right\} .
\end{gathered}
$$


Thus

$$
\left[r_{1} r_{2}\right] \leq M_{g}^{-1}\left[\exp \left\{\left({ }_{v_{2}} \bar{\sigma}_{g}+\varepsilon\right)\left[r_{1} r_{2}\right]^{{ }_{2} \rho_{g}}\right\}\right]
$$

and

$$
M_{g}^{-1}\left(r_{1}, r_{2}\right) \geq\left[\left(\frac{\log \left(r_{1} r_{2}\right)}{\left(v_{2} \bar{\sigma}_{g}-\varepsilon\right)}\right)^{\frac{1}{v_{2} \rho_{g}}}\right] .
$$

From the definitions of $v_{2} \bar{\tau}_{f}$ and $v_{2} \tau_{f}$, we have for all sufficiently large values of $r_{1}, r_{2}$ that

$$
\begin{aligned}
& M_{f}\left(r_{1}, r_{2}\right) \leq \exp \left[\left(v_{2} \bar{\tau}_{f}+\varepsilon\right)\left[r_{1} r_{2}\right]^{v_{2}} \lambda_{f}\right], \\
& M_{f}\left(r_{1}, r_{2}\right) \geq \exp \left[\left(v_{2} \tau_{f}-\varepsilon\right)\left[r_{1} r_{2}\right]^{v_{2}} \lambda_{f}\right]
\end{aligned}
$$

and also for a sequence of values of $r_{1}, r_{2}$ tending to infinity, we get that

$$
\begin{aligned}
& M_{f}\left(r_{1}, r_{2}\right) \geq \exp \left[\left(v_{2} \bar{\tau}_{f}-\varepsilon\right)\left[r_{1} r_{2}\right]^{v_{2}} \lambda_{f}\right], \\
& M_{f}\left(r_{1}, r_{2}\right) \leq \exp \left[\left(v_{2} \tau_{f}+\varepsilon\right)\left[r_{1} r_{2}\right]^{v_{2}} \lambda_{f}\right] .
\end{aligned}
$$

Similarly from the definitions of $v_{2} \bar{\tau}_{g}$ and $v_{2} \tau_{g}$, it follows for all sufficiently large values of $r_{1}, r_{2}$ that

$$
M_{g}\left(r_{1}, r_{2}\right) \leq \exp \left[\left({ }_{v_{2}} \bar{\tau}_{g}+\varepsilon\right) \cdot\left[r_{1} r_{2}\right]^{v_{2}} \lambda_{g}\right] .
$$

Thus

$$
\left[r_{1} r_{2}\right] \leq M_{g}^{-1}\left[\exp \left[\left({ }_{v_{2}} \bar{\tau}_{g}+\varepsilon\right) \cdot\left[r_{1} r_{2}\right]^{v_{2}} \lambda_{g}\right]\right]
$$

and

$$
\begin{gathered}
M_{g}^{-1}\left(r_{1}, r_{2}\right) \geq\left[\left(\frac{\log \left(r_{1} r_{2}\right)}{\left(v_{2} \bar{\tau}_{g}+\varepsilon\right)}\right)^{\frac{1}{v_{2} \lambda_{g}}}\right] . \\
M_{g}\left(r_{1}, r_{2}\right) \geq \exp \left[\left({ }_{v_{2}} \tau_{g}-\varepsilon\right) \cdot\left[r_{1} r_{2}\right]^{{ }_{2}} \lambda_{g}\right] .
\end{gathered}
$$

Thus

$$
\left[r_{1} r_{2}\right] \geq M_{g}^{-1}\left[\exp \left[\left({ }_{v_{2}} \tau_{g}-\varepsilon\right) \cdot\left[r_{1} r_{2}\right]^{v_{2}} \lambda_{g}\right]\right]
$$

and

$$
M_{g}^{-1}\left(r_{1}, r_{2}\right) \leq\left[\left(\frac{\log \left(r_{1} r_{2}\right)}{\left(v_{2} \tau_{g}-\varepsilon\right)}\right)^{\frac{1}{v_{2} \lambda_{g}}}\right] .
$$

and for a sequence of values of $r_{1}, r_{2}$ tending to infinity, we obtain that

$$
M_{g}\left(r_{1}, r_{2}\right) \geq \exp \left[\left({ }_{v_{2}} \bar{\tau}_{g}-\varepsilon\right) \cdot\left[r_{1} r_{2}\right]^{v_{2}} \lambda_{g}\right]
$$


that is

$$
\left[r_{1} r_{2}\right] \geq M_{g}^{-1}\left[\exp \left[\left(v_{2} \bar{\tau}_{g}-\varepsilon\right) \cdot\left[r_{1} r_{2}\right]^{{ }^{v_{2}}} \lambda_{g}\right]\right]
$$

and

$$
\begin{gathered}
M_{g}^{-1}\left(r_{1}, r_{2}\right) \leq\left[\left(\frac{\log \left(r_{1} r_{2}\right)}{\left(v_{2} \bar{\tau}_{g}-\varepsilon\right)}\right)^{\frac{1}{v_{2} \lambda_{g}}}\right] \\
M_{g}\left(r_{1}, r_{2}\right) \leq \exp \left[\left({ }_{v_{2}} \tau_{g}+\varepsilon\right) \cdot\left[r_{1} r_{2}\right]^{v_{2}} \lambda_{g}\right],
\end{gathered}
$$

that is

$$
\left[r_{1} r_{2}\right] \leq M_{g}^{-1}\left[\exp \left[\left({ }_{v_{2}} \tau_{g}+\varepsilon\right) \cdot\left[r_{1} r_{2}\right]^{v_{2}} \lambda_{g}\right]\right]
$$

and

$$
M_{g}^{-1}\left(r_{1}, r_{2}\right) \geq\left[\left(\frac{\log \left(r_{1} r_{2}\right)}{\left(v_{2} \tau_{g}-\varepsilon\right)}\right)^{\frac{1}{v_{2} \lambda_{g}}}\right]
$$

Now from (3) and in view of (13), we get for a sequence of values of $r_{1}, r_{2}$ tending to infinity that

$$
M_{g}^{-1} M_{f}\left(r_{1}, r_{2}\right) \geq M_{g}^{-1}\left[\exp \left[\left(v_{2} \sigma_{f}-\varepsilon\right)\left[r_{1} r_{2}\right]^{v_{2}} \rho_{f}\right]\right] .
$$

Thus

$$
M_{g}^{-1} M_{f}\left(r_{1}, r_{2}\right) \geq\left[\left(\frac{\log \exp \left[\left(v_{2} \sigma_{f}-\varepsilon\right)\left[r_{1} r_{2}\right]^{\nu_{2}} \rho_{f}\right]}{\left({ }_{2} \bar{\tau}_{g}+\varepsilon\right)}\right)^{\frac{1}{v_{2} \lambda_{g}}}\right]
$$

that is

$$
M_{g}^{-1} M_{f}\left(r_{1}, r_{2}\right) \geq\left[\frac{\left({ }_{v_{2}} \sigma_{f}-\varepsilon\right)}{\left(v_{2} \bar{\tau}_{g}+\varepsilon\right)}\right]^{\frac{1}{v_{2} \lambda_{g}}} \cdot\left[r_{1} r_{2}\right]^{\frac{v_{2} \rho_{f}}{v_{2} \lambda_{g}}}
$$

and

$$
\frac{M_{g}^{-1} M_{f}\left(r_{1}, r_{2}\right)}{\left[r_{1} r_{2}\right]^{\frac{v_{2} \rho_{f}}{v_{2} \lambda_{g}}}} \geq\left[\frac{\left({ }_{v_{2}} \sigma_{f}-\varepsilon\right)}{\left({ }_{v_{2}} \bar{\tau}_{g}+\varepsilon\right)}\right]^{\frac{1}{\lambda_{g}}}
$$

Since in view of Lemma $1, \frac{v_{2} \rho_{f}}{v_{2} \lambda_{g}} \geq{ }_{v_{2}} \rho_{g}(f)$ and as $\varepsilon(>0)$ is arbitrary, therefore it follows from above that

$$
\limsup _{r_{1}, r_{2} \rightarrow \infty} \frac{M_{g}^{-1} M_{f}\left(r_{1}, r_{2}\right)}{\left[r_{1} r_{2}\right]^{v_{2} \rho_{g}(f)}} \geq\left[\frac{v_{2} \sigma_{f}}{v_{2} \bar{\tau}_{g}}\right]^{\frac{1}{v_{2} \lambda_{g}}}
$$

that is

$$
v_{2} \sigma_{g}(f) \geq\left[\frac{v_{2} \sigma_{f}}{v_{2} \bar{\tau}_{g}}\right]^{\frac{1}{v_{2} \lambda_{g}}}
$$

Similarly from (2) and in view of (16), it follows for a sequence of values of $r_{1}, r_{2}$ tending to infinity that

$$
M_{g}^{-1} M_{f}\left(r_{1}, r_{2}\right) \geq M_{g}^{-1}\left[\exp \left[\left(v_{2} \bar{\sigma}_{f}-\varepsilon\right)\left[r_{1} r_{2}\right]^{v_{2}} \rho_{f}\right]\right]
$$


therefore

$$
M_{g}^{-1} M_{f}\left(r_{1}, r_{2}\right) \geq\left[\left(\frac{\log \exp \left[\left({ }_{v_{2}} \bar{\sigma}_{f}-\varepsilon\right)\left[r_{1} r_{2}\right]^{v_{2}} \rho_{f}\right]}{\left({ }_{v_{2}} \tau_{g}-\varepsilon\right)}\right)^{\frac{1}{v_{2} \lambda_{g}}}\right]
$$

that is

$$
M_{g}^{-1} M_{f}\left(r_{1}, r_{2}\right) \geq\left[\frac{\left(v_{2} \bar{\sigma}_{f}-\varepsilon\right)}{\left(v_{2} \tau_{g}+\varepsilon\right)}\right]^{\frac{1}{v_{2} \lambda_{g}}} \cdot\left[r_{1} r_{2}\right]^{\frac{v_{2} \rho_{f}}{v_{2} \lambda_{g}}}
$$

and

$$
\frac{M_{g}^{-1} M_{f}\left(r_{1}, r_{2}\right)}{\left[r_{1} r_{2}\right]^{\frac{v_{2} \rho_{f}}{v_{2}}}} \geq\left[\frac{\left({ }_{v_{2}} \bar{\sigma}_{f}-\varepsilon\right)}{\left(v_{2} \tau_{g}+\varepsilon\right)}\right]^{\frac{1}{v_{2} \lambda_{g}}}
$$

Since in view of Lemma 1, it follows that $\frac{v_{2} \rho_{f}}{v_{2} \lambda_{g}} \geq{ }_{v_{2}} \rho_{g}(f)$. Also $\varepsilon(>0)$ is arbitrary, so we get from above that

$$
\limsup _{r_{1}, r_{2} \rightarrow \infty} \frac{M_{g}^{-1} M_{f}\left(r_{1}, r_{2}\right)}{\left[r_{1} r_{2}\right]^{v_{2} \rho_{g}(f)}} \geq\left[\frac{v_{2} \bar{\sigma}_{f}}{v_{2} \tau_{g}}\right]^{\frac{1}{v_{2} \lambda_{g}}}
$$

and

$$
v_{2} \sigma_{g}(f) \geq\left[\frac{v_{2} \bar{\sigma}_{f}}{v_{2}} \tau_{g}^{\frac{1}{v_{2} \lambda_{g}}}\right.
$$

Again in view of (14), we have from (9) for all sufficiently large values of $r_{1}, r_{2}$ that

$$
M_{g}^{-1} M_{f}\left(r_{1}, r_{2}\right) \leq M_{g}^{-1}\left[\exp \left[\left(v_{2} \bar{\tau}_{f}+\varepsilon\right)\left[r_{1} r_{2}\right]^{v_{2}} \lambda_{f}\right]\right],
$$

therefore

$$
M_{g}^{-1} M_{f}\left(r_{1}, r_{2}\right) \leq\left[\left(\frac{\log \exp \left[\left({ }_{v_{2}} \bar{\tau}_{f}+\varepsilon\right)\left[r_{1} r_{2}\right]^{v_{2}} \lambda_{f}\right]}{\left(v_{2} \tau_{g}-\varepsilon\right)}\right)^{\frac{1}{v_{2} \lambda_{g}}}\right]
$$

that is

$$
M_{g}^{-1} M_{f}\left(r_{1}, r_{2}\right) \leq\left[\frac{\left(v_{2} \bar{\tau}_{f}+\varepsilon\right)}{\left(v_{2} \tau_{g}-\varepsilon\right)}\right]^{\frac{1}{v_{2} \lambda_{g}}} \cdot\left[r_{1} r_{2}\right]^{\frac{v_{2} \lambda_{f}}{v_{2} \lambda_{g}}}
$$

and

$$
\frac{M_{g}^{-1} M_{f}\left(r_{1}, r_{2}\right)}{\left[r_{1} r_{2}\right]^{\frac{v_{2} \lambda_{f}}{v_{2} \lambda_{g}}}} \leq\left[\frac{\left(v_{2} \bar{\tau}_{f}+\varepsilon\right)}{\left(v_{2} \tau_{g}-\varepsilon\right)}\right]^{\frac{1}{v_{2} \lambda_{g}}} .
$$

Since in view of Lemma 1, we get that $\frac{v_{2} \lambda_{f}}{v_{2} \lambda_{g}} \leq_{v_{2}} \rho_{g}(f)$ and as $\varepsilon(>0)$ is arbitrary, therefore it follows from above that

$$
\limsup _{r_{1}, r_{2} \rightarrow \infty} \frac{M_{g}^{-1} M_{f}\left(r_{1}, r_{2}\right)}{\left[r_{1} r_{2}\right]^{v_{2} \rho g}(f)} \leq\left[\frac{v_{2} \bar{\tau}_{f}}{v_{2} \tau_{g}}\right]^{\frac{1}{v_{2} \lambda_{g}}}
$$


and

$$
v_{2} \sigma_{g}(f) \leq\left[\frac{v_{2} \bar{\tau}_{f}}{v_{2} \tau_{g}}\right]^{\frac{1}{v_{2} \lambda_{g}}}
$$

Again in view of (6), we have from (1) for all sufficiently large values of $r_{1}, r_{2}$ that

$$
M_{g}^{-1} M_{f}\left(r_{1}, r_{2}\right) \leq M_{g}^{-1}\left[\exp \left[\left({ }_{v_{2}} \sigma_{f}+\varepsilon\right)\left[r_{1} r_{2}\right]^{v_{2}} \rho_{f}\right]\right]
$$

therefore

$$
M_{g}^{-1} M_{f}\left(r_{1}, r_{2}\right) \leq\left[\left(\frac{\log \exp \left[\left(v_{2} \sigma_{f}+\varepsilon\right)\left[r_{1} r_{2}\right]^{v_{2} \rho_{f}}\right]}{\left(v_{2} \bar{\sigma}_{g}-\varepsilon\right)}\right)^{\frac{1}{v_{2} \rho_{g}}}\right]
$$

that is

$$
M_{g}^{-1} M_{f}\left(r_{1}, r_{2}\right) \leq\left[\frac{\left(v_{2} \sigma_{f}+\varepsilon\right)}{\left(v_{2} \bar{\sigma}_{g}-\varepsilon\right)}\right]^{\frac{1}{v_{2} \rho_{g}}} \cdot\left[r_{1} r_{2}\right]^{\frac{v_{2} \rho_{f}}{v_{2} \rho_{g}}}
$$

and

$$
\frac{M_{g}^{-1} M_{f}\left(r_{1}, r_{2}\right)}{\left[r_{1} r_{2}\right]^{\frac{v_{2} \rho_{f}}{v_{g}}}} \leq\left[\frac{\left(v_{2} \sigma_{f}+\varepsilon\right)}{\left(v_{2} \bar{\sigma}_{g}-\varepsilon\right)}\right]^{\frac{1}{v_{2} \rho_{g}}} .
$$

As in view of Lemma 1, it follows that $\frac{v_{2} \rho_{f}}{v_{2} \rho_{g}} \leq{ }_{v_{2}} \rho_{g}(f)$. Since $\varepsilon(>0)$ is arbitrary, we get from (20) that

$$
\limsup _{r_{1}, r_{2} \rightarrow \infty} \frac{M_{g}^{-1} M_{f}\left(r_{1}, r_{2}\right)}{\left[r_{1} r_{2}\right]^{v_{2} \rho_{g}(f)}} \leq\left[\frac{v_{2} \sigma_{f}}{v_{2} \bar{\sigma}_{g}}\right]^{\frac{1}{v_{2} \rho_{g}}}
$$

that is

$$
v_{2} \sigma_{g}(f) \leq\left[\frac{v_{2} \sigma_{f}}{v_{2} \bar{\sigma}_{g}}\right]^{\frac{1}{v_{2} \rho_{g}}}
$$

Further in view of (6), we have from (9) for all sufficiently large values of $r_{1}, r_{2}$ that

$$
M_{g}^{-1} M_{f}\left(r_{1}, r_{2}\right) \leq M_{g}^{-1}\left[\exp \left[\left(v_{2} \bar{\tau}_{f}+\varepsilon\right)\left[r_{1} r_{2}\right]^{v_{2}} \lambda_{f}\right]\right]
$$

therefore

$$
M_{g}^{-1} M_{f}\left(r_{1}, r_{2}\right) \leq\left[\left(\frac{\log \exp \left[\left({ }_{v_{2}} \bar{\tau}_{f}+\varepsilon\right)\left[r_{1} r_{2}\right]^{v_{2}} \lambda_{f}\right]}{\left(v_{2} \bar{\sigma}_{g}-\varepsilon\right)}\right)^{\frac{1}{v_{2} \rho_{g}}}\right]
$$

that is

$$
M_{g}^{-1} M_{f}\left(r_{1}, r_{2}\right) \leq\left[\frac{\left(v_{2} \bar{\tau}_{f}+\varepsilon\right)}{\left({ }_{v_{2}} \bar{\sigma}_{g}-\varepsilon\right)}\right]^{\frac{1}{\rho_{g}}} \cdot\left[r_{1} r_{2}\right]^{\frac{v_{2} \lambda_{f}}{v_{2} \rho_{g}}}
$$


and

$$
\frac{M_{g}^{-1} M_{f}\left(r_{1}, r_{2}\right)}{\left[r_{1} r_{2}\right]^{\frac{v_{2}}{v_{2} \rho_{g}}}} \leq\left[\frac{\left({ }_{v_{2}} \bar{\tau}_{f}+\varepsilon\right)}{\left(v_{2} \bar{\sigma}_{g}-\varepsilon\right)}\right]^{\frac{1}{v_{2} \rho_{g}}} .
$$

Since in view of Lemma 1, we get that $\frac{v_{2} \lambda_{f}}{v_{2} \rho_{g}} \leq{ }_{v_{2}} \rho_{g}(f)$ and as $\varepsilon(>0)$ is arbitrary, therefore it follows from above that

$$
\limsup _{r_{1}, r_{2} \rightarrow \infty} \frac{M_{g}^{-1} M_{f}\left(r_{1}, r_{2}\right)}{\left[r_{1} r_{2}\right]^{v_{2} \rho_{g}^{*}(f)}} \leq\left[\frac{v_{2} \bar{\tau}_{f}}{v_{2} \bar{\sigma}_{g}}\right]^{\frac{1}{v_{2} \rho_{g}}}
$$

that is

$$
{ }_{v_{2}} \sigma_{g}^{L^{*}}(f) \leq\left[\frac{v_{2} \bar{\tau}_{f}}{v_{2} \bar{\sigma}_{g}}\right]^{\frac{1}{v_{2} \rho_{g}}} .
$$

Thus the first part of the theorem follows from (17), (18), (19), (21) and (22).

Further from (2) and in view of (13), we get for all sufficiently large values of $r_{1}, r_{2}$ that

$$
M_{g}^{-1} M_{f}\left(r_{1}, r_{2}\right) \geq M_{g}^{-1}\left[\exp \left[\left(v_{2} \bar{\sigma}_{f}-\varepsilon\right)\left[r_{1} r_{2}\right]^{v_{2}} \rho_{f}\right]\right]
$$

and

$$
M_{g}^{-1} M_{f}\left(r_{1}, r_{2}\right) \geq\left[\left(\frac{\log \exp \left[\left({ }_{v_{2}} \bar{\sigma}_{f}-\varepsilon\right)\left[r_{1} r_{2}\right]^{v_{2} \rho_{f}}\right]}{\left({ }_{v_{2}} \bar{\tau}_{g}+\varepsilon\right)}\right)^{\frac{1}{v_{2} \lambda_{g}}}\right]
$$

therefore

$$
M_{g}^{-1} M_{f}\left(r_{1}, r_{2}\right) \geq\left[\frac{\left({ }_{v_{2}} \bar{\sigma}_{f}-\varepsilon\right)}{\left(v_{2} \bar{\tau}_{g}+\varepsilon\right)}\right]^{\frac{1}{v_{2} \lambda_{g}}} \cdot\left[r_{1} r_{2}\right]^{\frac{v_{2} \rho_{f}}{2_{2} \lambda_{g}}}
$$

and

$$
\frac{M_{g}^{-1} M_{f}\left(r_{1}, r_{2}\right)}{\left[r_{1} r_{2}\right]^{\frac{v_{2} \rho_{f}}{v_{g}}}} \geq\left[\frac{\left({ }_{v_{2}} \bar{\sigma}_{f}-\varepsilon\right)}{\left(v_{2} \bar{\tau}_{g}+\varepsilon\right)}\right]^{\frac{1}{v_{2} \lambda_{g}}}
$$

Now in view of Lemma 1, it follows that $\frac{v_{2} \rho_{f}}{v_{2} \lambda_{g}} \geq{ }_{v_{2}} \rho_{g}(f)$. Since $\varepsilon(>0)$ is arbitrary, we get from above that

$$
\liminf _{r_{1}, r_{2} \rightarrow \infty} \frac{M_{g}^{-1} M_{f}\left(r_{1}, r_{2}\right)}{\left[r_{1} r_{2}\right]^{v_{2} \rho_{g}(f)}} \geq\left[\frac{v_{2} \bar{\sigma}_{f}}{v_{2} \bar{\tau}_{g}}\right]^{\frac{1}{v_{2} \lambda_{g}}}
$$

and

$$
v_{2} \bar{\sigma}_{g}(f) \geq\left[\frac{v_{2} \bar{\sigma}_{f}}{v_{2} \bar{\tau}_{g}}\right]^{\frac{1}{v_{2} \lambda_{g}}}
$$

Also in view of (7), we get from (1) for a sequence of values of $r_{1}, r_{2}$ tending to infinity that

$$
M_{g}^{-1} M_{f}\left(r_{1}, r_{2}\right) \leq M_{g}^{-1}\left[\exp \left[\left(v_{2} \sigma_{f}+\varepsilon\right)\left[r_{1} r_{2}\right]^{v_{2}} \rho_{f}\right]\right],
$$


therefore

$$
M_{g}^{-1} M_{f}\left(r_{1}, r_{2}\right) \leq\left[\left(\frac{\log \exp \left[\left({ }_{v_{2}} \sigma_{f}+\varepsilon\right)\left[r_{1} r_{2}\right]_{2}^{v_{2} \rho_{f}}\right]}{\left(v_{2} \sigma_{g}-\varepsilon\right)}\right)^{\frac{1}{v_{2} \rho_{g}}}\right]
$$

that is

$$
M_{g}^{-1} M_{f}\left(r_{1}, r_{2}\right) \leq\left[\frac{\left({ }_{v_{2}} \sigma_{f}+\varepsilon\right)}{\left({ }_{v_{2}} \sigma_{g}-\varepsilon\right)}\right]^{\frac{1}{v_{2} \rho_{g}}} \cdot\left[r_{1} r_{2}\right]^{\frac{v_{2} \rho_{f}}{v_{2} \rho_{g}}}
$$

and

$$
\frac{M_{g}^{-1} M_{f}\left(r_{1}, r_{2}\right)}{\left[r_{1} r_{2}\right]^{\frac{v_{2} \rho_{f}}{v_{2} \rho_{g}}}} \leq\left[\frac{\left({ }_{v_{2}} \sigma_{f}+\varepsilon\right)}{\left({ }_{v_{2}} \sigma_{g}-\varepsilon\right)}\right]^{\frac{1}{v_{2} \rho_{g}(m, p)}}
$$

Again in view of Lemma 1, $\frac{v_{2} \rho_{f}}{v_{2} \rho_{g}} \leq{ }_{{ }_{2}} \rho_{g}(f)$ and $\varepsilon(>0)$ is arbitrary, so we get from (24) that

$$
\liminf _{r_{1}, r_{2} \rightarrow \infty} \frac{M_{g}^{-1} M_{f}\left(r_{1}, r_{2}\right)}{\left[r_{1} r_{2}\right]^{v_{2} \rho_{g}(f)}} \leq\left[\frac{v_{2} \sigma_{f}}{v_{2} \sigma_{g}}\right]^{\frac{1}{v_{2} \rho_{g}}}
$$

that is

$$
v_{2} \bar{\sigma}_{g}(f) \leq\left[\frac{{ }_{v_{2}} \sigma_{f}}{{ }_{v_{2}} \sigma_{g}}\right]^{\frac{1}{v_{2} \rho_{g}}}
$$

Likewise from (4) and in view of (6), it follows for a sequence of values of $r_{1}, r_{2}$ tending to infinity that

$$
M_{g}^{-1} M_{f}\left(r_{1}, r_{2}\right) \leq M_{g}^{-1}\left[\exp \left[\left(v_{2} \bar{\sigma}_{f}+\varepsilon\right)\left[r_{1} r_{2}\right]^{v_{2}} \rho_{f}\right]\right],
$$

thefore

$$
M_{g}^{-1} M_{f}\left(r_{1}, r_{2}\right) \leq\left[\left(\frac{\log \exp \left[\left(v_{2} \bar{\sigma}_{f}+\varepsilon\right)\left[r_{1} r_{2}\right]^{v_{2} \rho_{f}^{L^{*}}}\right]}{\left(v_{2} \bar{\sigma}_{g}-\varepsilon\right)}\right)^{\frac{1}{v_{2} \rho_{g}}}\right]
$$

that is

$$
M_{g}^{-1} M_{f}\left(r_{1}, r_{2}\right) \leq\left[\frac{\left(v_{2} \bar{\sigma}_{f}+\varepsilon\right)}{\left({ }_{v_{2}} \bar{\sigma}_{g}-\varepsilon\right)}\right]^{\frac{1}{v_{2} \rho_{g}}} \cdot\left[r_{1} r_{2}\right]^{\frac{v_{2} \rho_{f}}{v_{2} \rho_{g}}}
$$

and

$$
\frac{M_{g}^{-1} M_{f}\left(r_{1}, r_{2}\right)}{\left[r_{1} r_{2}\right]^{\frac{v_{2} \rho_{f}}{v_{2}} \rho_{g}}} \leq\left[\frac{\left({ }_{v_{2}} \bar{\sigma}_{f}+\varepsilon\right)}{\left({ }_{v_{2}} \bar{\sigma}_{g}-\varepsilon\right)}\right]^{\frac{1}{v_{2} \rho_{g}}} .
$$

Analogously, we get from (26) that

$$
\liminf _{r_{1}, r_{2} \rightarrow \infty} \frac{M_{g}^{-1} M_{f}\left(r_{1}, r_{2}\right)}{\left[r_{1} r_{2}\right]^{\rho_{g}(f)}} \leq\left[\frac{v_{2} \bar{\sigma}_{f}}{v_{2} \bar{\sigma}_{g}}\right]^{\frac{1}{v_{2} \rho_{g}}}
$$


and

$$
v_{2} \bar{\sigma}_{g}(f) \leq\left[\frac{v_{2} \bar{\sigma}_{f}}{v_{2} \bar{\sigma}_{g}}\right]^{\frac{1}{v_{2} \rho_{g}}}
$$

since in view of Lemma $1, \frac{v_{2} \rho_{f}}{v_{2} \rho_{g}} \leq{ }_{v_{2}} \rho_{g}(f)$ and $\varepsilon(>0)$ is arbitrary.

Further in view of (15), we get from (9) for a sequence of values of $r_{1}, r_{2}$ tending to infinity that

$$
M_{g}^{-1} M_{f}\left(r_{1}, r_{2}\right) \leq M_{g}^{-1}\left[\exp \left[\left(v_{2} \bar{\tau}_{f}+\varepsilon\right)\left[r_{1} r_{2}\right]^{v_{2}} \lambda_{f}\right]\right]
$$

thefore

$$
M_{g}^{-1} M_{f}\left(r_{1}, r_{2}\right) \leq\left[\left(\frac{\log \exp \left[\left({ }_{v_{2}} \bar{\tau}_{f}+\varepsilon\right)\left[r_{1} r_{2}\right]^{v_{2}} \lambda_{f}\right]}{\left.{ }_{v_{2}} \bar{\tau}_{g}-\varepsilon\right)}\right)^{\frac{1}{v_{2} \lambda_{g}}}\right]
$$

that is

$$
M_{g}^{-1} M_{f}\left(r_{1}, r_{2}\right) \leq\left[\frac{\left(v_{2} \bar{\tau}_{f}+\varepsilon\right)}{\left({ }_{v_{2}} \bar{\tau}_{g}-\varepsilon\right)}\right]^{\frac{1}{v_{2} \lambda_{g}}} \cdot\left[r_{1} r_{2}\right]^{\frac{v_{2} \lambda_{f}}{v_{2} \lambda_{g}}}
$$

and

$$
\frac{M_{g}^{-1} M_{f}\left(r_{1}, r_{2}\right)}{\left[r_{1} r_{2}\right]^{\frac{v_{2} \lambda_{f}}{v_{2} \lambda_{g}}}} \leq\left[\frac{\left(v_{2} \bar{\tau}_{f}+\varepsilon\right)}{\left(v_{2} \bar{\tau}_{g}-\varepsilon\right)}\right]^{\frac{1}{v_{2} \lambda_{g}}} .
$$

As in view of Lemma 1, we get that $\frac{v_{2} \lambda_{f}}{v_{2} \lambda_{g}} \leq{ }_{v_{2}} \rho_{g}(f)$ and as $\varepsilon(>0)$ is arbitrary, it follows from above that

$$
\liminf _{r_{1}, r_{2} \rightarrow \infty} \frac{M_{g}^{-1} M_{f}\left(r_{1}, r_{2}\right)}{\left[r_{1} r_{2}\right]^{v_{2} \rho_{g}^{*}(f)}} \leq\left[\frac{v_{2} \bar{\tau}_{f}}{v_{2} \bar{\tau}_{g}}\right]^{\frac{1}{v_{2} \lambda_{g}}}
$$

that is

$$
v_{2} \bar{\sigma}_{g}^{L^{*}}(f) \leq\left[\frac{v_{2} \bar{\tau}_{f}}{v_{2} \bar{\tau}_{g}}\right]^{\frac{1}{v_{2} \lambda_{g}}} .
$$

Similarly from (12) and in view of (14), it follows for a sequence of values of $r_{1}, r_{2}$ tending to infinity that

$$
M_{g}^{-1} M_{f}\left(r_{1}, r_{2}\right) \leq M_{g}^{-1}\left[\exp \left[\left({ }_{2} \tau_{f}+\varepsilon\right)\left[r_{1} r_{2}\right]^{v_{2}} \lambda_{g}\right]\right],
$$

therefore

$$
M_{g}^{-1} M_{f}\left(r_{1}, r_{2}\right) \leq\left[\left(\frac{\log \exp \left[\left({ }_{v_{2}} \tau_{f}+\varepsilon\right)\left[r_{1} r_{2}\right]^{v_{2}} \lambda_{f}\right]}{\left({ }_{v_{2}} \tau_{g}-\varepsilon\right)}\right)^{\frac{1}{v_{2} \lambda_{g}}}\right]
$$

that is

$$
M_{g}^{-1} M_{f}\left(r_{1}, r_{2}\right) \leq\left[\frac{\left(v_{2} \tau_{f}+\varepsilon\right)}{\left(v_{2} \tau_{g}-\varepsilon\right)}\right]^{\frac{1}{v_{2} \lambda_{g}}} \cdot\left[r_{1} r_{2}\right]^{\frac{v_{2} \lambda_{f}}{v_{2} \lambda_{g}}}
$$


and

$$
\frac{M_{g}^{-1} M_{f}\left(r_{1}, r_{2}\right)}{\left[r_{1} r_{2}\right]^{\frac{v_{2}}{v_{2} \lambda_{g}}}} \leq\left[\frac{\left({ }_{v_{2}} \tau_{f}+\varepsilon\right)}{\left(v_{2} \tau_{g}-\varepsilon\right)}\right]^{\frac{1}{v_{2} \lambda_{g}}} .
$$

Also in view of Lemma 1, we get that $\frac{v_{2} \lambda_{f}}{v_{2} \lambda_{g}} \leq{ }_{v_{2}} \rho_{g}(f)$ and as $\varepsilon(>0)$ is arbitrary, therefore it follows from above that

$$
\liminf _{r_{1}, r_{2} \rightarrow \infty} \frac{M_{g}^{-1} M_{f}\left(r_{1}, r_{2}\right)}{\left[r_{1} r_{2}\right]^{v_{2} \rho_{g}(f)}} \leq\left[\frac{v_{2} \tau_{f}}{v_{2} \tau_{g}}\right]^{\frac{1}{v_{2} \lambda_{g}}}
$$

that is

$$
{ }_{v_{2}} \bar{\sigma}_{g}(f) \leq\left[\frac{v_{2} \tau_{f}}{v_{2}} \tau_{g}\right]^{\frac{1}{v_{2} \lambda_{g}}}
$$

Again in view of (7), we get from (9) for a sequence of values of $r_{1}, r_{2}$ tending to infinity that

$$
M_{g}^{-1} M_{f}\left(r_{1}, r_{2}\right) \leq M_{g}^{-1}\left[\exp \left[\left(v_{2} \bar{\tau}_{f}+\varepsilon\right)\left[r_{1} r_{2}\right]^{v_{2}} \lambda_{f}\right]\right],
$$

therefore

$$
M_{g}^{-1} M_{f}\left(r_{1}, r_{2}\right) \leq\left[\left(\frac{\log \exp \left[\left({ }_{v_{2}} \bar{\tau}_{f}+\varepsilon\right)\left[r_{1} r_{2}\right]^{v_{2}} \lambda_{f}\right]}{\left(v_{2} \sigma_{g}-\varepsilon\right)}\right)^{\frac{1}{v_{2} \rho_{g}}}\right]
$$

that is

$$
M_{g}^{-1} M_{f}\left(r_{1}, r_{2}\right) \leq\left[\frac{\left({ }_{v_{2}} \bar{\tau}_{f}+\varepsilon\right)}{\left({ }_{v_{2}} \sigma_{g}-\varepsilon\right)}\right]^{\frac{1}{v_{2} \rho_{g}}} \cdot\left[r_{1} r_{2}\right]^{\frac{v_{2} \lambda_{f}}{v_{2} \rho_{g}}}
$$

and

$$
\frac{M_{g}^{-1} M_{f}\left(r_{1}, r_{2}\right)}{\left[r_{1} r_{2}\right]^{\frac{v_{2}}{v_{2} \rho^{*}}}} \leq\left[\frac{\left(v_{2} \bar{\tau}_{f}+\varepsilon\right)}{v_{2}\left(\sigma_{g}-\varepsilon\right)}\right]^{\frac{1}{v_{2} \rho_{g}}}
$$

Since in view of Lemma 1, we get that $\frac{v_{2} \lambda_{f}}{v_{2} \rho_{g}} \leq{ }_{v_{2}} \rho_{g}(f)$ and as $\varepsilon(>0)$ is arbitrary, it follows from above that

$$
\liminf _{r_{1}, r_{2} \rightarrow \infty} \frac{M_{g}^{-1} M_{f}\left(r_{1}, r_{2}\right)}{\left[r_{1} r_{2}\right]^{v_{2} \rho_{g}^{*}(f)}} \leq\left[\frac{v_{2} \bar{\tau}_{f}}{v_{2} \sigma_{g}}\right]^{\frac{1}{v_{2} \rho_{g}}}
$$

that is

$$
v_{2} \bar{\sigma}_{g}(f) \leq\left[\frac{v_{2} \bar{\tau}_{f}}{{ }_{v_{2}} \sigma_{g}}\right]^{\frac{1}{v_{2} \rho_{g}}} .
$$

Similarly from (12) and in view of (6), it follows for a sequence of values of $r_{1}, r_{2}$ tending to infinity that

$$
M_{g}^{-1} M_{f}\left(r_{1}, r_{2}\right) \leq M_{g}^{-1}\left[\exp \left[\left(v_{2} \tau_{f}+\varepsilon\right)\left[r_{1} r_{2}\right]^{v_{2}} \lambda_{f}\right]\right],
$$


therefore

$$
M_{g}^{-1} M_{f}\left(r_{1}, r_{2}\right) \leq\left[\left(\frac{\log \exp \left[\left(v_{2} \tau_{f}+\varepsilon\right)\left[r_{1} r_{2}\right]^{v_{2}} \lambda_{f}\right]}{\left(v_{2} \bar{\sigma}_{g}-\varepsilon\right)}\right)^{\frac{1}{v_{2} \rho_{g}}}\right]
$$

that is

$$
M_{g}^{-1} M_{f}\left(r_{1}, r_{2}\right) \leq\left[\frac{\left(v_{2} \tau_{f}+\varepsilon\right)}{\left(v_{2} \bar{\sigma}_{g}-\varepsilon\right)}\right]^{\frac{1}{v_{2} \rho_{g}}} \cdot\left[r_{1} r_{2}\right]^{\frac{v_{2} \lambda_{f}}{v_{2} \rho_{g}}}
$$

and

$$
\frac{M_{g}^{-1} M_{f}\left(r_{1}, r_{2}\right)}{\left[r_{1} r_{2}\right]^{\frac{v_{2}}{v_{f}} \rho_{g}}} \leq\left[\frac{\left(v_{2} \tau_{f}+\varepsilon\right)}{\left(v_{2} \bar{\sigma}_{g}-\varepsilon\right)}\right]^{\frac{1}{v_{2} \rho_{g}}} .
$$

As in view of Lemma 1, we get that $\frac{{ }_{v_{2}} \lambda_{f}}{v_{2} \rho_{g}} \leq{ }_{v_{2}} \rho_{g}(f)$ and as $\varepsilon(>0)$ is arbitrary, therefore it follows from above that

$$
\liminf _{r_{1}, r_{2} \rightarrow \infty} \frac{M_{g}^{-1} M_{f}\left(r_{1}, r_{2}\right)}{\left[r_{1} r_{2}\right]^{v_{2} \rho_{g}(f)}} \leq\left[\frac{v_{2} \tau_{f}}{v_{2} \bar{\sigma}_{g}}\right]^{\frac{1}{v_{2} \rho_{g}}}
$$

that is

$$
v_{2} \bar{\sigma}_{g}(f) \leq\left[\frac{v_{2} \tau_{f}}{v_{2} \bar{\sigma}_{g}}\right]^{\frac{1}{v_{2} \rho_{g}}}
$$

Hence the second part of the theorem follows from (23), (25), (27), (28), (29), (30) and (31).

Theorem 2. Let $f\left(z_{1}, z_{2}\right)$ and $g\left(z_{1}, z_{2}\right)$ be any two entire functions with $0 \leq{ }_{v_{2}} \lambda_{f} \leq{ }_{v_{2}} \rho_{f}<\infty$ and $0 \leq{ }_{v_{2}} \lambda_{g} \leq{ }_{v_{2}} \rho_{g}<\infty$. Then

$$
\max \left\{\begin{array}{l}
{\left[\frac{v_{2} \bar{\tau}_{f}}{v_{2} \bar{\tau}_{g}}\right]^{\frac{1}{v_{2} \lambda_{g}}},\left[\frac{v_{2} \tau_{f}}{v_{2} \tau_{g}}\right]^{\frac{1}{v_{2} \lambda_{g}}},\left[\frac{v_{2} \bar{\sigma}_{f}}{v_{2} \bar{\sigma}_{g}}\right]^{\frac{1}{v_{2} \rho_{g}}},} \\
{\left[\frac{v_{2} \sigma_{f}}{v_{2} \sigma_{g}}\right]^{\frac{1}{v_{2} \rho_{g}}},\left[\frac{v_{2} \sigma_{f}}{v_{2} \bar{\tau}_{g}}\right]^{\frac{1}{v_{2} \lambda_{g}}},\left[\frac{v_{2} \bar{\sigma}_{f}}{v_{2} \tau_{g}}\right]^{\frac{1}{v_{2} \lambda_{g}}}}
\end{array}\right\} \leq v_{v_{2}} \bar{\tau}_{g}(f) \leq\left[\frac{v_{2} \bar{\tau}_{f}}{v_{2} \bar{\sigma}_{g}}\right]^{\frac{1}{v_{2} \rho_{g}}}
$$

and

$$
\max \left\{\left[\frac{v_{2} \bar{\sigma}_{f}}{v_{2} \sigma_{g}}\right]^{\frac{1}{v_{2} \rho_{g}}},\left[\frac{v_{2} \tau_{f}}{v_{2}} \bar{\tau}_{g}\right]^{\frac{1}{v_{2} \lambda_{g}}},\left[\frac{v_{2} \bar{\sigma}_{f}}{v_{2} \bar{\tau}_{g}}\right]^{\frac{1}{v_{2} \lambda_{g}}}\right\} \leq v_{v_{2}} \tau_{g}(f) \leq \min \left\{\left[\frac{v_{2} \tau_{f}}{v_{2} \bar{\sigma}_{g}}\right]^{\frac{1}{v_{2} \rho_{g}}},\left[\frac{v_{2} \bar{\tau}_{f}}{v_{2} \sigma_{g}}\right]^{\frac{1}{v_{2} \rho_{g}}}\right\} .
$$

Proof. We obtain from (11) and (13), for a sequence of values of $r_{1}, r_{2}$ tending to infinity that

$$
M_{g}^{-1} M_{f}\left(r_{1}, r_{2}\right) \geq M_{g}^{-1}\left[\exp \left[\left(v_{2} \bar{\tau}_{f}-\varepsilon\right)\left[r_{1} r_{2}\right]^{v_{2}} \lambda_{f}\right]\right]
$$

therefore

$$
M_{g}^{-1} M_{f}\left(r_{1}, r_{2}\right) \geq\left[\left(\frac{\log \exp \left[\left(v_{2} \bar{\tau}_{f}-\varepsilon\right)\left[r_{1} r_{2}\right]^{v_{2}} \lambda_{f}\right]}{\left.{ }_{v_{2}} \bar{\tau}_{g}+\varepsilon\right)}\right)^{\frac{1}{v_{2} \lambda_{g}}}\right]
$$


that is

$$
M_{g}^{-1} M_{f}\left(r_{1}, r_{2}\right) \geq\left[\frac{\left(v_{2} \bar{\tau}_{f}-\varepsilon\right)}{\left(\bar{\tau}_{g}+\varepsilon\right)}\right]^{\frac{1}{v_{2} \lambda_{g}}} \cdot\left[r_{1} r_{2}\right]^{\frac{v_{2} \lambda_{f}}{v_{2} \lambda_{g}}}
$$

and

$$
\frac{M_{g}^{-1} M_{f}\left(r_{1}, r_{2}\right)}{\left[r_{1} r_{2}\right]^{\frac{v_{2} \lambda_{f}}{v_{2} \lambda_{g}}}} \geq\left[\frac{\left(v_{2} \bar{\tau}_{f}-\varepsilon\right)}{\left(v_{2} \bar{\tau}_{g}+\varepsilon\right)}\right]^{\frac{1}{\lambda_{g}}} .
$$

Since in view of Lemma 1, we get that $\frac{v_{2} \lambda_{f}}{v_{2} \lambda_{g}} \geq{ }_{v_{2}} \lambda_{g}(f)$ and as $\varepsilon(>0)$ is arbitrary, it follows from above that

$$
\limsup _{r_{1}, r_{2} \rightarrow \infty} \frac{M_{g}^{-1} M_{f}\left(r_{1}, r_{2}\right)}{\left[r_{1} r_{2}\right]^{v_{2} \lambda_{g}(f)}} \geq\left[\frac{v_{2} \bar{\tau}_{f}}{v_{2} \bar{\tau}_{g}}\right]^{\frac{1}{v_{2} \lambda_{g}}}
$$

and

$$
v_{2} \bar{\tau}_{g}(f) \geq\left[\frac{v_{2} \bar{\tau}_{f}}{v_{2} \bar{\tau}_{g}}\right]^{\frac{1}{v_{2} \lambda_{g}}}
$$

Further we obtain from (10) and (16), for a sequence of values of $r_{1}, r_{2}$ tending to infinity that

$$
M_{g}^{-1} M_{f}\left(r_{1}, r_{2}\right) \geq M_{g}^{-1}\left[\exp \left[\left(v_{2} \tau_{f}-\varepsilon\right)\left[r_{1} r_{2}\right]^{v_{2}} \lambda_{f}\right]\right],
$$

therefore

$$
M_{g}^{-1} M_{f}\left(r_{1}, r_{2}\right) \geq\left[\left(\frac{\log \exp \left[\left({ }_{v_{2}} \tau_{f}-\varepsilon\right)\left[r_{1} r_{2}\right]^{v_{2} \lambda_{f}}\right]}{\left({ }_{2} \tau_{g}-\varepsilon\right)}\right)^{\frac{1}{v_{2} \lambda_{g}}}\right]
$$

that is

$$
M_{g}^{-1} M_{f}\left(r_{1}, r_{2}\right) \geq\left[\frac{\left({ }_{2} \tau_{f}-\varepsilon\right)}{\left({ }_{2} \tau_{g}-\varepsilon\right)}\right]^{\frac{1}{v_{2} \lambda_{g}}} \cdot\left[r_{1} r_{2}\right]^{\frac{v_{2} \lambda_{f}}{v_{2} \lambda_{g}}}
$$

and

$$
\frac{M_{g}^{-1} M_{f}\left(r_{1}, r_{2}\right)}{\left[r_{1} r_{2}\right]^{\frac{v_{2} \lambda_{f}}{v_{2} \lambda_{g}}}} \geq\left[\frac{\left(v_{2} \tau_{f}-\varepsilon\right)}{\left(v_{2} \tau_{g}-\varepsilon\right)}\right]^{\frac{1}{v_{2} \lambda_{g}}}
$$

As in view of Lemma 1 , we get that $\frac{v_{2} \lambda_{f}}{v_{2} \lambda_{g}} \geq{ }_{v_{2}} \lambda_{g}(f)$ and as $\varepsilon(>0)$ is arbitrary, it follows from above that

$$
\limsup _{r_{1}, r_{2} \rightarrow \infty} \frac{M_{g}^{-1} M_{f}\left(r_{1}, r_{2}\right)}{\left[r_{1} r_{2}\right]^{v_{2} \lambda_{g}(f)}} \geq\left[\frac{v_{2} \tau_{f}}{v_{2} \tau_{g}}\right]^{\frac{1}{v_{2} \lambda_{g}}}
$$


and

$$
v_{2} \bar{\tau}_{g}(f) \geq\left[\frac{v_{2} \tau_{f}}{v_{2} \tau_{g}}\right]^{\frac{1}{v_{2} \lambda_{g}}}
$$

Now from (3) and in view of (5), we get for a sequence of values of $r_{1}, r_{2}$ tending to infinity that

$$
M_{g}^{-1} M_{f}\left(r_{1}, r_{2}\right) \geq M_{g}^{-1}\left[\exp \left[\left(v_{2} \sigma_{f}-\varepsilon\right)\left[r_{1} r_{2}\right]^{v_{2}} \rho_{f}\right]\right],
$$

therefore

$$
M_{g}^{-1} M_{f}\left(r_{1}, r_{2}\right) \geq\left[\left(\frac{\log \exp \left[\left(v_{2} \sigma_{f}-\varepsilon\right)\left[r_{1} r_{2}\right]_{2}^{\nu_{2} \rho_{f}}\right]}{\left(v_{2} \sigma_{g}+\varepsilon\right)}\right)^{\frac{1}{v_{2} \rho_{g}}}\right]
$$

that is

$$
M_{g}^{-1} M_{f}\left(r_{1}, r_{2}\right) \geq\left[\frac{\left({ }_{v_{2}} \sigma_{f}-\varepsilon\right)}{\left({ }_{v_{2}} \sigma_{g}+\varepsilon\right)}\right]^{\frac{1}{v_{2} \rho_{g}}} \cdot\left[r_{1} r_{2}\right]^{\frac{v_{2} \rho_{f}}{v_{2} \rho_{g}}}
$$

and

$$
\frac{M_{g}^{-1} M_{f}\left(r_{1}, r_{2}\right)}{\left[r_{1} r_{2}\right]^{\frac{v_{2} \rho_{f}}{v_{2}}}} \geq\left[\frac{\left({ }_{v_{2}} \sigma_{f}-\varepsilon\right)}{\left(v_{2} \sigma_{g}+\varepsilon\right)}\right]^{\frac{1}{v_{2} \rho_{g}}} .
$$

Also from (2) and in view of (8), it follows for a sequence of values of $r_{1}, r_{2}$ tending to infinity that

$$
M_{g}^{-1} M_{f}\left(r_{1}, r_{2}\right) \geq M_{g}^{-1}\left[\exp \left[\left(v_{2} \bar{\sigma}_{f}-\varepsilon\right)\left[r_{1} r_{2}\right]^{{ }_{2}} \rho_{f}\right]\right],
$$

therefore

$$
M_{g}^{-1} M_{f}\left(r_{1}, r_{2}\right) \geq\left[\left(\frac{\log \exp \left[\left({ }_{v_{2}} \bar{\sigma}_{f}-\varepsilon\right)\left[r_{1} r_{2}\right]^{v_{2}} \rho_{f}\right]}{\left(v_{2} \bar{\sigma}_{g}-\varepsilon\right)}\right)^{\frac{1}{v_{2} \rho_{g}}}\right]
$$

that is

$$
M_{g}^{-1} M_{f}\left(r_{1}, r_{2}\right) \geq\left[\frac{\left({ }_{v_{2}} \bar{\sigma}_{f}-\varepsilon\right)}{\left({ }_{v_{2}} \bar{\sigma}_{g}+\varepsilon\right)}\right]^{\frac{1}{v_{2} \rho_{g}}} \cdot\left[r_{1} r_{2}\right]^{\frac{v_{2} \rho_{f}}{v_{2} \rho_{g}}}
$$

and

$$
\frac{M_{g}^{-1} M_{f}\left(r_{1}, r_{2}\right)}{\left[r_{1} r_{2}\right]^{\frac{v_{2} \rho_{f}}{v_{g}}}} \geq\left[\frac{\left(v_{2} \bar{\sigma}_{f}-\varepsilon\right)}{\left(v_{2} \bar{\sigma}_{g}+\varepsilon\right)}\right]^{\frac{1}{v_{2} \rho_{g}}}
$$

As in view of Lemma $1, \frac{v_{2} \rho_{f}}{v_{2} \rho_{g}} \geq{ }_{v_{2}} \lambda_{g}(f)$ and $\varepsilon(>0)$ is arbitrary, we get from (34) that

$$
\limsup _{r_{1}, r_{2} \rightarrow \infty} \frac{M_{g}^{-1} M_{f}\left(r_{1}, r_{2}\right)}{\left[r_{1} r_{2}\right]^{v_{2}} \lambda_{g}(f)} \geq\left[\frac{v_{2} \sigma_{f}}{v_{2} \sigma_{g}}\right]^{\frac{1}{v_{2} \rho_{g}}}
$$


that is

$$
v_{2} \bar{\tau}_{g}(f) \geq\left[\frac{{ }_{v_{2}} \sigma_{f}}{{ }_{v_{2}} \sigma_{g}}\right]^{\frac{1}{v_{2} \rho g}}
$$

Simialrly, we get from (35) that

$$
\limsup _{r_{1}, r_{2} \rightarrow \infty} \frac{M_{g}^{-1} M_{f}\left(r_{1}, r_{2}\right)}{\left[r_{1} r_{2}\right]^{\lambda_{g}(f)}} \geq\left[\frac{\bar{\sigma}_{f}}{\bar{\sigma}_{g}}\right]^{\frac{1}{\rho_{g}}}
$$

that is

$$
\bar{\tau}_{g}(f) \geq\left[\frac{\bar{\sigma}_{f}}{\bar{\sigma}_{g}}\right]^{\frac{1}{\rho_{g}}}
$$

since in view of Lemma $1, \frac{v_{2} \rho_{f}}{v_{2} \rho_{g}} \leq{ }_{v_{2}} \lambda_{g}(f)$ and $\varepsilon(>0)$ is arbitrary.

Likewise from (3) and in view of (13), we get for a sequence of values of $r_{1}, r_{2}$ tending to infinity that

$$
M_{g}^{-1} M_{f}\left(r_{1}, r_{2}\right) \geq M_{g}^{-1}\left[\exp \left[\left(v_{2} \sigma_{f}-\varepsilon\right)\left[r_{1} r_{2}\right]^{{ }_{2}} \rho_{f}\right]\right],
$$

therefore

$$
M_{g}^{-1} M_{f}\left(r_{1}, r_{2}\right) \geq\left[\left(\frac{\log \exp \left[\left(v_{2} \sigma_{f}-\varepsilon\right)\left[r_{1} r_{2}\right]^{v_{2}} \rho_{f}\right]}{\left(v_{2} \bar{\tau}_{g}+\varepsilon\right)}\right)^{\frac{1}{v_{2} \lambda_{g}}}\right]
$$

that is

$$
M_{g}^{-1} M_{f}\left(r_{1}, r_{2}\right) \geq\left[\frac{\left({ }_{2} \sigma_{f}-\varepsilon\right)}{\left(v_{2} \bar{\tau}_{g}+\varepsilon\right)}\right]^{\frac{1}{v_{2} \lambda_{g}}} \cdot\left[r_{1} r_{2}\right]^{\frac{{ }_{2} \rho_{f}}{v_{2} \lambda_{g}}}
$$

and

$$
\frac{M_{g}^{-1} M_{f}\left(r_{1}, r_{2}\right)}{\left[r_{1} r_{2}\right]^{\frac{v_{2} \rho_{f}}{v_{2} \lambda_{g}}}} \geq\left[\frac{\left({ }_{v_{2}} \sigma_{f}-\varepsilon\right)}{\left(v_{2} \bar{\tau}_{g}+\varepsilon\right)}\right]^{\frac{1}{v_{2} \lambda_{g}}}
$$

Since in view of Lemma 1, we get that $\frac{{ }_{v_{2}} \rho_{f}}{v_{2} \lambda_{g}} \geq{ }_{v_{2}} \lambda_{g}(f)$ and as $\varepsilon(>0)$ is arbitrary, it follows from above that

$$
\limsup _{r_{1}, r_{2} \rightarrow \infty} \frac{M_{g}^{-1} M_{f}\left(r_{1}, r_{2}\right)}{\left[r_{1} r_{2}\right]^{\lambda_{g}(f)}} \geq\left[\frac{v_{2} \sigma_{f}}{v_{2} \bar{\tau}_{g}}\right]^{\frac{1}{v_{2} \lambda_{g}}}
$$

that is

$$
v_{2} \bar{\tau}_{g}(f) \geq\left[\frac{v_{2} \sigma_{f}}{{ }_{v_{2}} \bar{\tau}_{g}}\right]^{\frac{1}{v_{2} \lambda_{g}}} .
$$

Further from (2) and in view of (16), it follows for a sequence of values of $r_{1}, r_{2}$ tending to infinity that

$$
M_{g}^{-1} M_{f}\left(r_{1}, r_{2}\right) \geq M_{g}^{-1}\left[\exp \left[\left(v_{2} \bar{\sigma}_{f}-\varepsilon\right)\left[r_{1} r_{2}\right]^{v_{2}} \rho_{f}\right]\right],
$$


therefore

$$
M_{g}^{-1} M_{f}\left(r_{1}, r_{2}\right) \geq\left[\left(\frac{\log \exp \left[\left({ }_{v_{2}} \bar{\sigma}_{f}-\varepsilon\right)\left[r_{1} r_{2}\right]_{2} \rho_{f} \rho_{f}\right.}{\left(v_{2} \tau_{g}-\varepsilon\right)}\right)^{\frac{1}{v_{2} \lambda_{g}}}\right]
$$

that is

$$
M_{g}^{-1} M_{f}\left(r_{1}, r_{2}\right) \geq\left[\frac{\left(v_{2} \bar{\sigma}_{f}-\varepsilon\right)}{\left(v_{2} \tau_{g}-\varepsilon\right)}\right]^{\frac{1}{v_{2} \lambda_{g}}} \cdot\left[r_{1} r_{2}\right]^{\frac{v_{2} \rho_{f}}{v_{2} \lambda_{g}}}
$$

and

$$
\frac{M_{g}^{-1} M_{f}\left(r_{1}, r_{2}\right)}{\left[r_{1} r_{2}\right]^{\frac{v_{2} \rho_{f}}{v_{2} \lambda_{g}}}} \geq\left[\frac{\left({ }_{v_{2}} \bar{\sigma}_{f}-\varepsilon\right)}{\left(v_{2} \tau_{g}-\varepsilon\right)}\right]^{\frac{1}{v_{2} \lambda_{g}}} .
$$

As in view of Lemma 1, we get that $\frac{\rho_{f}}{v_{v_{2}} \lambda_{g}} \geq{ }_{v_{2}} \lambda_{g}(f)$ and as $\varepsilon(>0)$ is arbitrary, therefore it follows from above that

$$
\limsup _{r_{1}, r_{2} \rightarrow \infty} \frac{M_{g}^{-1} M_{f}\left(r_{1}, r_{2}\right)}{\left[r_{1} r_{2}\right]^{\lambda_{g}(f)}} \geq\left[\frac{v_{2} \bar{\sigma}_{f}}{v_{2} \tau_{g}}\right]^{\frac{1}{v_{2} \lambda_{g}}}
$$

that is

$$
v_{2} \bar{\tau}_{g}(f) \geq\left[\frac{v_{2} \bar{\sigma}_{f}}{v_{2} \tau_{g}}\right]^{\frac{1}{v_{2} \lambda_{g}}} .
$$

Again from (6) and (9), we have for all sufficiently large values of $r_{1}, r_{2}$ that

$$
M_{g}^{-1} M_{f}\left(r_{1}, r_{2}\right) \leq M_{g}^{-1}\left[\exp \left[\left(v_{2} \bar{\tau}_{f}+\varepsilon\right)\left[r_{1} r_{2}\right]^{v_{2}} \lambda_{f}\right]\right]
$$

therefore

$$
M_{g}^{-1} M_{f}\left(r_{1}, r_{2}\right) \leq\left[\left(\frac{\log \exp \left[\left({ }_{v_{2}} \bar{\tau}_{f}+\varepsilon\right)\left[r_{1} r_{2}\right]^{v_{2}} \lambda_{f}\right]}{\left({ }_{v_{2}} \bar{\sigma}_{g}-\varepsilon\right)}\right)^{\frac{1}{v_{2} \rho_{g}}}\right]
$$

that is

$$
M_{g}^{-1} M_{f}\left(r_{1}, r_{2}\right) \leq\left[\frac{\left(v_{2} \bar{\tau}_{f}+\varepsilon\right)}{\left({ }_{v_{2}} \bar{\sigma}_{g}-\varepsilon\right)}\right]^{\frac{1}{v_{2} \rho_{g}}} \cdot\left[r_{1} r_{2}\right]^{\frac{v_{2} \lambda_{f}}{v_{2} \rho_{g}}}
$$

and

$$
\frac{M_{g}^{-1} M_{f}\left(r_{1}, r_{2}\right)}{\left[r_{1} r_{2}\right]^{\frac{v_{2}}{v_{2} \rho_{g}}}} \leq\left[\frac{\left(v_{2} \bar{\tau}_{f}+\varepsilon\right)}{\left(v_{2} \bar{\sigma}_{g}-\varepsilon\right)}\right]^{\frac{1}{v_{2} \rho_{g}}}
$$

Since in view of Lemma 1, we get that $\frac{v_{2} \lambda_{f}}{v_{2} \rho_{g}} \leq{ }_{v_{2}} \lambda_{g}(f)$ and as $\varepsilon(>0)$ is arbitrary, therefore it follows from above that

$$
\limsup _{r_{1}, r_{2} \rightarrow \infty} \frac{M_{g}^{-1} M_{f}\left(r_{1}, r_{2}\right)}{\left[r_{1} r_{2}\right]^{v_{2}} \lambda_{g}(f)} \leq\left[\frac{v_{2} \bar{\tau}_{f}}{v_{2} \bar{\sigma}_{g}}\right]^{\frac{1}{v_{2} \rho_{g}}}
$$


that is

$$
v_{2} \bar{\tau}_{g}(f) \leq\left[\frac{v_{2} \bar{\tau}_{f}}{v_{2} \bar{\sigma}_{g}}\right]^{\frac{1}{v_{2} \rho_{g}}} .
$$

Thus the first part of the theorem follows from (32), (33), (36), (37), (38), (39) and (40).

Further from (10) and in view of (13), we get for all sufficiently large values of $r_{1}, r_{2}$ that

$$
M_{g}^{-1} M_{f}\left(r_{1}, r_{2}\right) \geq M_{g}^{-1}\left[\exp \left[\left(v_{2} \tau_{f}-\varepsilon\right)\left[r_{1} r_{2}\right]^{v_{2}} \lambda_{f}\right]\right],
$$

therefore

$$
M_{g}^{-1} M_{f}\left(r_{1}, r_{2}\right) \geq\left[\left(\frac{\log \exp \left[\left({ }_{v_{2}} \tau_{f}-\varepsilon\right)\left[r_{1} r_{2}\right]^{{ }_{2}} \lambda_{f}\right]}{\left({ }_{v_{2}} \bar{\tau}_{g}+\varepsilon\right)}\right)^{\frac{1}{v_{2} \lambda_{g}}}\right]
$$

that is

$$
M_{g}^{-1} M_{f}\left(r_{1}, r_{2}\right) \geq\left[\frac{\left(v_{2} \tau_{f}-\varepsilon\right)}{\left({ }_{v_{2}} \bar{\tau}_{g}+\varepsilon\right)}\right]^{\frac{1}{v_{2} \lambda_{g}}} \cdot\left[r_{1} r_{2}\right]^{\frac{v_{2} \lambda_{f}}{v_{2} \lambda_{g}}}
$$

and

$$
\frac{M_{g}^{-1} M_{f}\left(r_{1}, r_{2}\right)}{\left[r_{1} r_{2}\right]^{\frac{v_{2} \lambda_{f}}{v_{2} \lambda_{g}}}} \geq\left[\frac{\left(v_{2} \tau_{f}-\varepsilon\right)}{\left(v_{2} \bar{\tau}_{g}+\varepsilon\right)}\right]^{\frac{1}{v_{2} \lambda_{g}}}
$$

Since in view of Lemma 1, we get that $\frac{v_{2} \lambda_{f}}{v_{2} \lambda_{g}} \geq{ }_{v_{2}} \lambda_{g}(f)$ and as $\varepsilon(>0)$ is arbitrary, therefore it follows from above that

$$
\liminf _{r_{1}, r_{2} \rightarrow \infty} \frac{M_{g}^{-1} M_{f}\left(r_{1}, r_{2}\right)}{\left[r_{1} r_{2}\right]^{v_{2} \lambda_{g}(f)}} \geq\left[\frac{v_{2} \tau_{f}}{v_{2}}\right]^{\frac{1}{v_{g} \lambda_{g}}}
$$

that is

$$
v_{2} \tau_{g}(f) \geq\left[\frac{v_{2} \tau_{f}}{v_{2} \bar{\tau}_{g}}\right]^{\frac{1}{v_{2} \lambda_{g}}}
$$

Again from (2) and in view of (5), we get for all sufficiently large values of $r_{1}, r_{2}$ that

$$
M_{g}^{-1} M_{f}\left(r_{1}, r_{2}\right) \geq M_{g}^{-1}\left[\exp \left[\left(v_{2} \bar{\sigma}_{f}-\varepsilon\right)\left[r_{1} r_{2}\right]^{v_{2}} \rho_{f}\right]\right],
$$

therefore

$$
M_{g}^{-1} M_{f}\left(r_{1}, r_{2}\right) \geq\left[\left(\frac{\log \exp \left[\left({ }_{v_{2}} \bar{\sigma}_{f}-\varepsilon\right)\left[r_{1} r_{2}\right]^{v_{2}} \rho_{f}\right]}{\left({ }_{v_{2}} \sigma_{g}+\varepsilon\right)}\right)^{\frac{1}{v_{2} \rho_{g}}}\right]
$$

that is

$$
M_{g}^{-1} M_{f}\left(r_{1}, r_{2}\right) \geq\left[\frac{\left({ }_{v_{2}} \bar{\sigma}_{f}-\varepsilon\right)}{\left({ }_{v_{2}} \sigma_{g}+\varepsilon\right)}\right]^{\frac{1}{v_{2} \rho_{g}}} \cdot\left[r_{1} r_{2}\right]^{\frac{v_{2} \rho_{f}}{v_{2} \rho_{g}}}
$$


and

$$
\frac{M_{g}^{-1} M_{f}\left(r_{1}, r_{2}\right)}{\left[r_{1} r_{2}\right]^{\frac{v_{2} \rho_{f}}{v_{g}}}} \geq\left[\frac{\left(v_{2} \bar{\sigma}_{f}-\varepsilon\right)}{\left(v_{2} \sigma_{g}+\varepsilon\right)}\right]^{\frac{1}{v_{2} \rho_{g}}} .
$$

As in view of Lemma $1, \frac{v_{2} \rho_{f}}{v_{2} \rho_{g}} \geq{ }_{v_{2}} \lambda_{g}(f)$ and $\varepsilon(>0)$ is arbitrary, we get from (42) that

$$
\liminf _{r_{1}, r_{2} \rightarrow \infty} \frac{M_{g}^{-1} M_{f}\left(r_{1}, r_{2}\right)}{\left[r_{1} r_{2}\right]^{v_{2} \lambda_{g}(f)}} \geq\left[\frac{v_{2} \bar{\sigma}_{f}}{v_{2} \sigma_{g}}\right]^{\frac{1}{v_{2} \rho_{g}}}
$$

that is

$$
v_{2} \tau_{g}(f) \geq\left[\frac{v_{2} \bar{\sigma}_{f}}{{ }_{v_{2}} \sigma_{g}}\right]^{\frac{1}{v_{2} \rho_{g}}} .
$$

Again from (2) and in view of (13), we get for all sufficiently large values of $r_{1}, r_{2}$ that

$$
M_{g}^{-1} M_{f}\left(r_{1}, r_{2}\right) \geq M_{g}^{-1}\left[\exp \left[\left(v_{2} \bar{\sigma}_{f}-\varepsilon\right)\left[r_{1} r_{2}\right]^{v_{2}} \rho_{f}\right]\right],
$$

therefore

$$
M_{g}^{-1} M_{f}\left(r_{1}, r_{2}\right) \geq\left[\left(\frac{\log \exp \left[\left(v_{2} \bar{\sigma}_{f}-\varepsilon\right)\left[r_{1} r_{2}\right]^{v_{2}} \rho_{f}\right]}{\left(v_{2} \bar{\tau}_{g}+\varepsilon\right)}\right)^{\frac{1}{v_{2} \lambda_{g}}}\right]
$$

that is

$$
M_{g}^{-1} M_{f}\left(r_{1}, r_{2}\right) \geq\left[\frac{\left({ }_{v_{2}} \bar{\sigma}_{f}-\varepsilon\right)}{\left(v_{2} \bar{\tau}_{g}+\varepsilon\right)}\right]^{\frac{1}{v_{2} \lambda_{g}}} \cdot\left[r_{1} r_{2}\right]^{\frac{v_{2} \rho_{f}}{v_{2} \lambda_{g}}}
$$

and

$$
\frac{M_{g}^{-1} M_{f}\left(r_{1}, r_{2}\right)}{\left[r_{1} r_{2}\right]^{\frac{v_{2} \rho_{f}}{v_{2} \lambda_{g}}}} \geq\left[\frac{\left(v_{2} \bar{\sigma}_{f}-\varepsilon\right)}{\left(v_{2} \bar{\tau}_{g}+\varepsilon\right)}\right]^{\frac{1}{v_{2} \lambda_{g}}}
$$

Since in view of Lemma 1, we get that $\frac{v_{2} \rho_{f}}{v_{2} \lambda_{g}} \geq{ }_{v_{2}} \lambda_{g}(f)$ and as $\varepsilon(>0)$ is arbitrary, therefore it follows from above that

$$
\liminf _{r_{1}, r_{2} \rightarrow \infty} \frac{M_{g}^{-1} M_{f}\left(r_{1}, r_{2}\right)}{\left[r_{1} r_{2}\right]^{v_{2} \lambda_{g}(f)}} \geq\left[\frac{v_{2} \bar{\sigma}_{f}}{v_{2} \bar{\tau}_{g}}\right]^{\frac{1}{v_{2} \lambda_{g}}}
$$

that is

$$
v_{2} \tau_{g}(f) \geq\left[\frac{v_{2} \bar{\sigma}_{f}}{v_{2} \bar{\tau}_{g}}\right]^{\frac{1}{v_{2} \lambda_{g}}} .
$$

Moreover, we get from (7) and (9) for a sequence of values of $r_{1}, r_{2}$ tending to infinity that

$$
M_{g}^{-1} M_{f}\left(r_{1}, r_{2}\right) \leq M_{g}^{-1}\left[\exp \left[\left(v_{2} \bar{\tau}_{f}+\varepsilon\right)\left[r_{1} r_{2}\right]^{v_{2}} \lambda_{f}\right]\right],
$$


therefore

$$
M_{g}^{-1} M_{f}\left(r_{1}, r_{2}\right) \leq\left[\left(\frac{\log \exp \left[\left({ }_{v_{2}} \bar{\tau}_{f}+\varepsilon\right)\left[r_{1} r_{2}\right]^{v_{2}} \lambda_{f}\right]}{\left({ }_{v_{2}} \sigma_{g}-\varepsilon\right)}\right)^{\frac{1}{v_{2} \rho_{g}}}\right]
$$

that is

$$
M_{g}^{-1} M_{f}\left(r_{1}, r_{2}\right) \leq\left[\frac{\left({ }_{v_{2}} \bar{\tau}_{f}+\varepsilon\right)}{\left({ }_{v_{2}} \sigma_{g}-\varepsilon\right)}\right]^{\frac{1}{v_{2} \rho_{g}}} \cdot\left[r_{1} r_{2}\right]^{\frac{v_{2} \lambda_{f}}{v_{2} \rho_{g}}}
$$

and

$$
\frac{M_{g}^{-1} M_{f}\left(r_{1}, r_{2}\right)}{\left[r_{1} r_{2}\right]^{\frac{v_{2}}{v_{2} \rho_{f}}}} \leq\left[\frac{\left(v_{2} \bar{\tau}_{f}+\varepsilon\right)}{\left(v_{2} \sigma_{g}-\varepsilon\right)}\right]^{\frac{1}{v_{2} \rho_{g}}}
$$

As in view of Lemma 1, we get that $\frac{v_{2} \lambda_{f}}{v_{2} \rho_{g}} \leq{ }_{v_{2}} \lambda_{g}(f)$ and as $\varepsilon(>0)$ is arbitrary, it follows from above that

$$
\liminf _{r_{1}, r_{2} \rightarrow \infty} \frac{M_{g}^{-1} M_{f}\left(r_{1}, r_{2}\right)}{\left[r_{1} r_{2}\right]^{v_{2}} \lambda_{g}(f)} \leq\left[\frac{v_{2} \bar{\tau}_{f}}{v_{2} \sigma_{g}}\right]^{\frac{1}{v_{2} \rho_{g}}}
$$

that is

$$
v_{2} \tau_{g}(f) \leq\left[\frac{v_{2} \bar{\tau}_{f}}{v_{2} \sigma_{g}}\right]^{\frac{1}{v_{2} \rho_{g}}}
$$

Similarly, from (12) and in view of (6), it follows for a sequence of values of $r_{1}, r_{2}$ tending to infinity that

$$
M_{g}^{-1} M_{f}\left(r_{1}, r_{2}\right) \leq M_{g}^{-1}\left[\exp \left[\left(v_{2} \tau_{f}+\varepsilon\right)\left[r_{1} r_{2}\right]^{{ }_{2}} \lambda_{f}\right]\right],
$$

therefore

$$
M_{g}^{-1} M_{f}\left(r_{1}, r_{2}\right) \leq\left[\left(\frac{\log \exp \left[\left(v_{2} \tau_{f}+\varepsilon\right)\left[r_{1} r_{2}\right]^{v_{2}} \lambda_{f}\right]}{\left(v_{2} \bar{\sigma}_{g}-\varepsilon\right)}\right)^{\frac{1}{v_{2} \rho_{g}}}\right]
$$

that is

$$
M_{g}^{-1} M_{f}\left(r_{1}, r_{2}\right) \leq\left[\frac{\left(v_{2} \tau_{f}+\varepsilon\right)}{\left({ }_{{ }_{2}} \bar{\sigma}_{g}-\varepsilon\right)}\right]^{\frac{1}{v_{2} \rho_{g}}} \cdot\left[r_{1} r_{2}\right]^{\frac{v_{2} \lambda_{f}}{v_{2} \rho_{g}}}
$$

and

$$
\frac{M_{g}^{-1} M_{f}\left(r_{1}, r_{2}\right)}{\left[r_{1} r_{2}\right]^{\frac{v_{2}}{v_{2}} \rho_{g}}} \leq\left[\frac{\left(v_{2} \tau_{f}+\varepsilon\right)}{\left(v_{2} \bar{\sigma}_{g}-\varepsilon\right)}\right]^{\frac{1}{v_{2} \rho_{g}}}
$$

Since in view of Lemma 1, we get that $\frac{v_{2} \lambda_{f}}{v_{2} \rho_{g}} \leq{ }_{v_{2}} \lambda_{g}(f)$ and as $\varepsilon(>0)$ is arbitrary, therefore it follows from above that

$$
\liminf _{r_{1}, r_{2} \rightarrow \infty} \frac{M_{g}^{-1} M_{f}\left(r_{1}, r_{2}\right)}{\left[r_{1} r_{2}\right]^{\lambda_{g}(f)}} \leq\left[\frac{v_{2} \tau_{f}}{v_{2} \bar{\sigma}_{g}}\right]^{\frac{1}{v_{2} \rho_{g}}}
$$


that is

$$
v_{2} \tau_{g}(f) \leq\left[\frac{v_{2} \tau_{f}}{{ }_{v_{2}} \bar{\sigma}_{g}}\right]^{\frac{1}{v_{2} \rho g}} .
$$

Hence the second part of the theorem follows from $(41),(43),(44),(45)$ and (46).

\section{Competing interests}

The authors declare that they have no competing interests.

\section{Authors' contributions}

All authors have contributed to all parts of the article. All authors read and approved the final manuscript.

\section{References}

[1] A. K. Agarwal : On the properties of entire function of two complex variables, Canadian Journal of Mathematics, Vol. 20 (1968), pp. 51-57.

[2] L. Bernal : Crecimiento relativo de funciones enteras. Contribución al estudio de lasfunciones enteras coníndice exponencial finito, Doctoral Dissertation, University of Seville, Spain, 1984.

[3] L. Bernal : Orden relative de crecimiento de funciones enteras, Collect. Math., Vol. 39 (1988), pp.209-229.

[4] D. Banerjee and R. K. Datta : Relative order of entire functions of two complex variables, International J. of Math. Sci. \& Engg. Appls. (IJMSEA), Vol. 1, No. 1 (2007), pp. 141-154

[5] S. K. Datta, T. Biswas and G. K. Mondal: A note on the relative order of entire functions of two complex variables, International Journal of Pure and Applied Mathematics (IJPAM), Vol. 101, No. 3 (2015), pp. 339-347.

[6] S. K. Datta, T. Biswas and S. Bhattacharyya: On relative type and weak type of entire functions of two complex variables, Facta Universititatis series: Mathematics and Informatics, Accepted for publication (2016) and to appear.

[7] A. B. Fuks : Theory of analytic functions of several complex variables, (1963), Moscow.

[8] S. Halvarsson : Growth properties of entire functions depending on a parameter, Annales Polonici Mathematici, Vol. 14, No.1 (1996), pp. 71-96.

[9] C. O. Kiselman : Order and type as measure of growth for convex or entire functions, Proc. Lond. Math. Soc., Vol. 66, No.3 (1993), pp. 152-186.

[10] C. O. Kiselman : Plurisubharmonic functions and potential theory in several complex variable, a contribution to the book project, Development of Mathematics, 1950-2000, edited by Hean-Paul Pier.

[11] B. K. Lahiri and D. Banerjee : A note on relative order of entire functions, Bull. Cal. Math. Soc. Vol. 97, No. 3 (2005), pp. $201-206$. 